\title{
ApneBoot: A Novel Device for Apnea Management in Premature Infants
}

\author{
Mona Sharma ${ }^{1 *}$, Triloki Keshri ${ }^{1}$, Sourabh Shetty ${ }^{1}$, Ratul Narain ${ }^{1}$ and Ranjan Pejaver ${ }^{2}$ \\ ${ }^{1}$ BEMPU Health Pvt. Ltd, Bangalore, India \\ ${ }^{2}$ Paediatrician \& Neonatologist, Meenakshi Hospital (People Tree Hospitals), Bangalore, India
}

*Corresponding author: Mona Sharma, BEMPU Health Pvt. Ltd. Alsa Glenridge, 3C, 32, Langford Rd, Bheemanna Garden, Shanti

Nagar, Bengaluru, Karnataka 560027, India

\section{ARTICLE INFO}

Received: 幽 April 30, 2020

Published: May 13, 2020

Citation: Mona S, Triloki K, Sourabh S, Ratul N, Ranjan P. ApneBoot: A Novel Device for Apnea Management in Premature Infants. Biomed J Sci \& Tech Res 27(4)-2020. BJSTR. MS.ID.004530.

Abbreviations: AOP: Apnea of Prematurity; PCM: Protection Circuit Module; DPPM: Dynamic Power Path Management; BD: Bradycardia and Desaturation; SNCU: Sick Newborn Care Unit; USAID: United States Agency for International Development;

\begin{abstract}
Apnea of Prematurity (AOP), defined as cessation of breathing lasting for more than 15 seconds and accompanied by hypoxia and/or brady cardia, is a serious risk factor for premature newborns. India has 3.5 million premature babies who are at risk of apneas every year. Prolonged apnea associated with hypoxia and bradycardia may contribute to hypoxic-ischemic injury of the immature brain. Research till date shows that higher frequency and severity of AOP are associated with a higher incidence of adverse outcomes or death, especially in low income settings. AOP is resolved by physical stimulation, often in the form of manual flicking of the baby's sole which triggers a nerve response to stimulate respiratory activity. Our innovation, ApneBoot, is designed to give an assured and instantaneous stimulation in response to an apnea by automating the standard of care flicking process. It alerts for bradycardia, hypoxia (using a built-in pulse oximeter) and resolves episodes of central apeas in newborns by instantaneous tactile stimulation to the foot sole. Devices currently available for managing neonatal apnea, are expensive; imported from high income countries; and only sound an alarm if no breathing is detected but do correct the apnea, making ApneBoot a novel device in the market. The pilot study for ApneBoot reports its accuracy as $94 \%$ for apnea detection and $70 \%$ for apnea resolution. Ongoing and future studies will look at the efficiency of ApneBoot in reducing the harmful consequences of prolonged hypoxia and also observe if it helps in reducing the workload of hospital personnel.
\end{abstract}

\section{Introduction}

In India, each year 3.5 million babies are born premature ranking it first among the top 10 countries with the greatest number of preterm births. Amongst these, approximately 361,600 children under five die due to direct preterm complications one of which is the Apnea of prematurity (AOP) [1]. Apnea of Prematurity (AOP) is defined as a 'cessation of breathing by the neonate for more than 15 seconds with hypoxia and/or bradycardia' [2]. Unresolved apneas are associated with hypoxia leading to other complications such as hypoxic-ischemic injury of the immature brain, impaired neuro development and even death [3]. Almost all infants born at $<29$ weeks gestation or $<1,000 \mathrm{~g}, 54 \%$ at 30 to 31 weeks, $15 \%$ at 32 to 33 weeks and $7 \%$ at 34 to 35 weeks gestation exhibit apnea, making the burden of AOP substantial [4]. AOP occurs in part due to poor nervous system coordination and physical stimulation, often in the form of manual flicking of the baby's sole, triggers a nerve response [5]. This excitatory nonspecific neuronal activity in the brainstem centre stimulates respiratory activity in the newborn. However, it is interesting to note that these stimulations only resolve central apneas and not obstructive apneas. Several studies have examined the therapeutic effects of mechanical stimulation [6] and found that tactile stimulations (manual or mechanical) shorten the duration of hypoxia, bradycardia and in most cases even prevents an apnea.

In low-resource settings, rapid intervention for apnea is difficult due to lack of vital sign monitors and skilled staff $(\approx 50 \%$ Indian SNCUs have inadequate nursing staff) [7]. These unresolved apneas may result in injury or death due to prolonged hypoxia. 
During informal visits conducted by BEMPU in several government hospitals across India, we observed that the lack of skilled staff and low nurse to patient ratio leads to sub-optimal management of apnea in newborns. These findings suggested a need for a device that not only detects and alerts but also resolves AOP in newborns in a timely manner. This auto-stimulation device may help in preventing apnea or hypoxia related injury or death in premature babies.

\section{ApneBoot: Device Description}

ApneBoot is a wearable device that alerts for bradycardia and hypoxia which are key symptoms of neonatal apnea in premature newborns. Further ApneBoot also resolves these alerted apnea episodes and prolonged hypoxia, which is done via an instantaneous tactile stimulation to the foot sole. The ApneBoot device is designed with a built-in pulse oximeter (with an alarm) and auto-stimulation mechanism that fits on the newborns foot. The device can be worn immediately upon birth and is designed to fit very small babies in the convenient form of a baby boot (Figure 1). The ApneBoot has an inbuilt algorithm which monitors for drops in oxygen saturation and/or heart rate. In the event of an episode, ApneBoot will;

1) Provide a vibro-tactile stimulus on the newborn's foot sole, which contains significant nerve endings. This stimulates the nervous system and restarts breathing.

2) It emits an audiovisual alarm to get the nurse's attention in case the apnea is secondary and requires interventions more than stimulation like assisted ventilation for resolution.

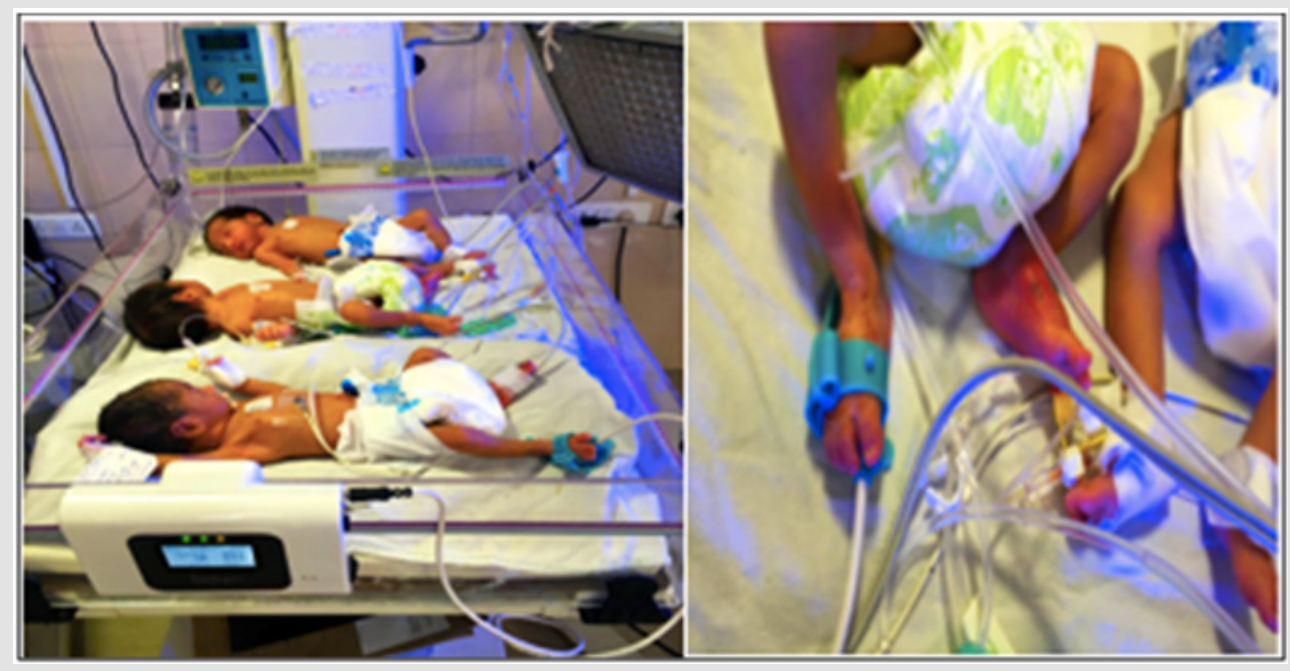

Figure 1: ApneBoot Device in the Neonatal Intensive Care Unit (NICU).

\section{The key Features For The Device Include (Figure 2)}

1) The device is reconfigurable to suit all newborns and gives rapid responses: To enhance the product usability and acceptability, the device has settings that are reconfigurable according to the doctor's recommendation. The device can be customized and used either only for monitoring and/ or stimulation. The stimulation threshold intensity can also be adjusted based on the newborn's weight\& age, medical conditions and the hospital preferences. It has been reported that the alarm sounds are distressing for the babies in the NICUs [8], necessitating alarm volume level feature in the present device.

2) It has a high accuracy built-in pulse oximeter that displays apnea duration, oxygen saturation and heart rate: ApneBoot has a medically approved Nonin pulse oximeter module proven to be accurate and safe as per the basic safety and essential performance of pulse oximeter equipment standard (ISO 80601-2-61:2011). This module has an accuracy of \pm 3 digits for measuring $\mathrm{SpO}_{2}$ in $70-100 \%$ range and \pm 3 digits for measuring Pulse Rate in 18-300 bpm range [9].
3) The device works continuously with electric power supply and also has a battery backup of 12 hours for long transport durations or under conditions of unreliable power supply: The Li-ion battery of $2900 \mathrm{mAh}$ used in ApneBoot is in compliance with secondary battery safety standard (EN 62133:2013). It has a feature of a protection circuit module (PCM) to avoid under-voltage and short circuit. The device features dynamic power path management (DPPM), which reduces the number of charge and discharge cycles on the battery, allowing for proper charge termination. This feature is most useful in lowresource settings where power failures are common with no battery back-ups and during transportation.

4) ApneBoot is safe, portable, robust, reusable and affordable making it an appropriate innovation for low-resource settings: The ApneBoot is evaluated for essential performance and basic safety under intended use cases. The risk management file is developed while designing the product as per EN IS014971:2012 and includes design mitigation features. The device is also tested as per IEC 60601-1-2:2007 $3^{\text {rd }}$ edition to ensure the electromagnetic compatibility of the device. 
ApneBoot software is written using state machine architecture to implement complex decision-making algorithms for Apnea detection and to respond rapidly to resolve Apnea before any injury can happen. The device is a light weight $(\sim 1 \mathrm{~kg})$ and easy to carry and designed keeping in mind the ease of use during transportation.

5) The device has a unique feature of tracking and displaying history of past bradycardia and hypoxia events that may help to identify recurring episodes and underlying causes like infections.

6) The device provides a vibro-tactile stimulation on the foot sole to trigger the central nervous system and restart breathing:
This stimulation mechanism is designed in the convenient form factor (using medical grade material) of a boot and includes two key mechanical parts i.e the sole-facing stimulating surface and the foot strap to firmly hold the boot to the foot. These parts are designed with ergonomics consideration for a firm grip to the sole while balancing comfort for the fragile skin of the newborns. For effective stimulation, the sole-facing surface of the boot was designed to have a smooth raised profile with multiple small sized bumps to act as concentration points. The boot strap has two length-adjusting straps (mid-foot strap\& the ankle strap) to hold the sole-facing stimulating surface firmly against the neonate's foot sole and prevent any lateral movement under the effect of vibration.

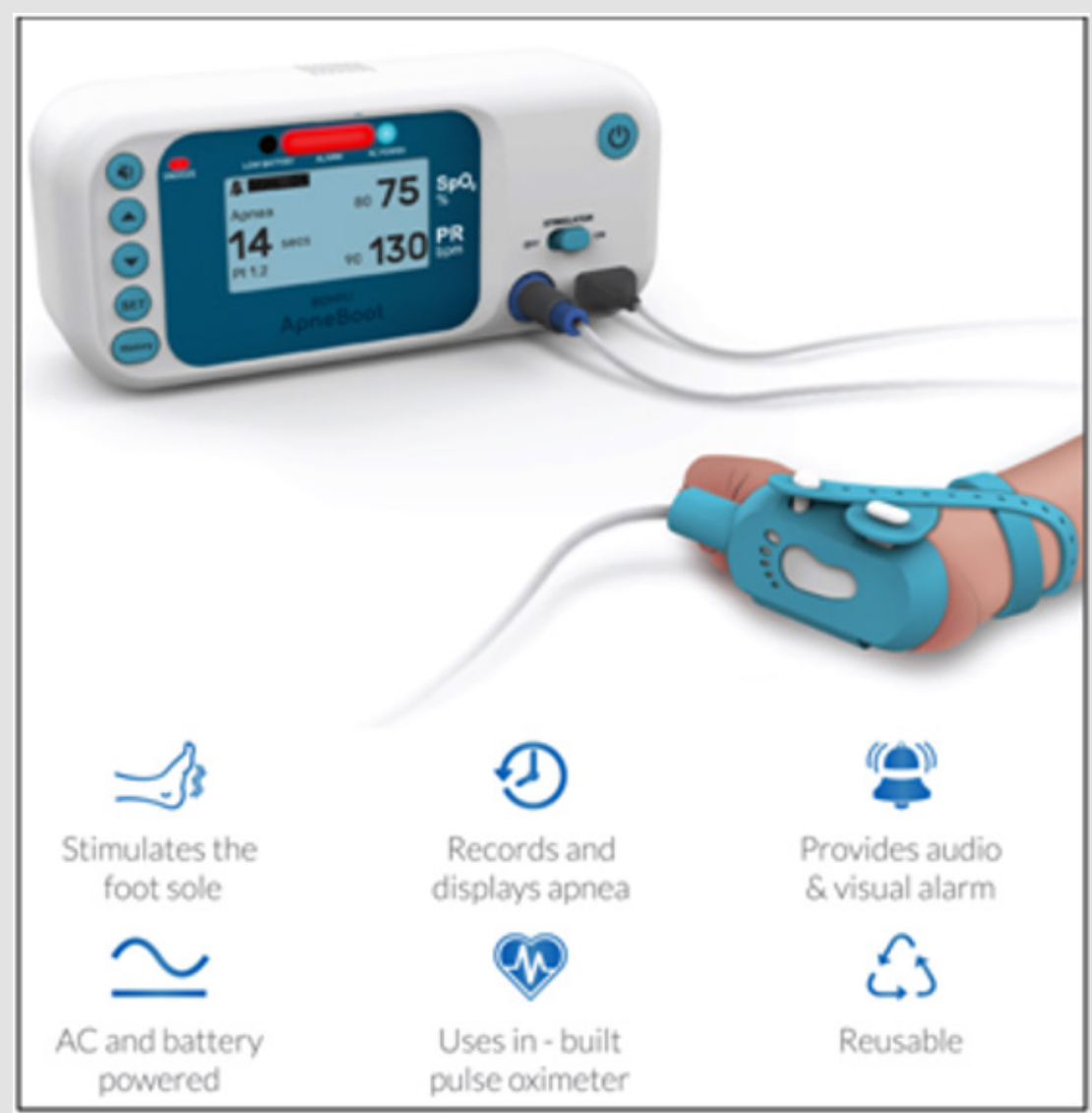

Figure 2: Key features of the ApneBoot Device.

ApneBoot has an edge over the routine pulse oximeters as it provides an intervention to resolves apneas. The dependency on the availability and prompt action by a nurse using only monitoring devices leaves babies vulnerable to risk of unattended apneas, especially in understaffed low resource settings. To the best of our knowledge, we have not come across a device which is an effective combination of pulse oximeter and apnea resolving devices available in the market. BEMPU's ApneBoot is specifically designed to meet the needs and overcome apnea correction in understaffed and under-equipped hospitals and ambulances.

\section{Clinical Evidence for ApneBoot}

ApneBoot is designed and developed with extensive user research with neonatologists and nurses working with preterm babies in NICU settings, especially of low-resource hospitals. A high quality FDA approved pulse oximeter module (OEM III) manufactured by Nonin Inc, USA is used as the built-in pulse oximeter to measure the $\mathrm{SpO}_{2}$ and $\mathrm{HR}$ making the device accurate, effective and safe. The initial prototype device was tested in-house for safety and accuracy. We conducted a small pilot study (with ethical permissions from the Institutional Ethical committee) 
to understand the need for stimulator boot dimensions and the stimulation intensity for the auto-stimulating boot in ApneBoot. The study recruited newborns (both female and males) ranging from 25 weeks to 36 weeks GA, weight ( 900 gms to 2000 gms) to gather information on the following foot measurements; instep girth, waist girth, ball girth, ankle girth, heel (big toe length, ball width). This information was utilised to design the perfect boot dimensions for effective stimulation and a comfortable grip. For the stimulation intensity, we initially calculated the adequate range of the auto-stimulation force by quantifying the force applied in stimulating respiration by 'flicking of sole' in neonates with apnea by various personnel including the nurses, doctors and controls. The stimulation intensity (from the above calculations), stimulation patterns and the number of cycles were finalized based on primary experiments done on the above recruited newborns.

Based on the findings, the stimulations found to restart breathing in the newborns most effectively and safely were included in the final design. Further, evidence was also collected of ApneBoot's ability to detect Bradycardia and Desaturation (BD), key symptoms of neonatal apnea and resolve apneas using the auto-stimulation. The final ApneBoot device thus uses an in-built algorithm that identifies BD events for any of 3 different conditions: 1) $\mathrm{SpO}_{2}<85 \%$ AND $\mathrm{HR}<100 \mathrm{bpm}$ for 5 seconds OR 2) $\mathrm{SpO}_{2}<75 \%$ for 5 seconds OR 3 ) $\mathrm{HR}<90 \mathrm{bpm}$ for 5 seconds. The final version of ApneBoot was thus hypothesised to accurately detect Bradycardia and Desaturation (BD) events related to apneas and further effectively auto-resolve and terminate apneic and hypoxic episodes through its tactile stimulating boot. To validate this hypothesis we conducted a prospective observational study at Niloufer Hospital, Hyderabad. The objectives of the pilot study were; 1 ) to measure the accuracy of a novel device in detecting Bradycardia and Desaturation (B\&D) events and 2) to determine its efficacy in resolving apneas in newborns with comparison to standard monitor (which only detects B\&D events and alerts).The study recruited $n=31$ preterm newborns ( $<34$ weeks of gestation) from the Sick Newborn Care Unit (SNCU), were provided with an ApneBoot device and monitored for a total of 47 days Enrolled newborns wearing an ApneBoot device were also connected to a standard monitor for comparison.

The study reports that ApneBoot positively predicts a B\&D event $94.1 \%$ times and falsely alarms $5.9 \%$ times as compared to the standard monitor. Visual confirmation showed 56/67 events to be positive apneas indicating $83.6 \%$ B\&D events coincided with apneas that were accurately picked up by ApneBoot. ApneBoot resolved 35 out of 50 events, making the device's efficacy of apnea resolution 70\%. The pilot study indicated Apne Boot's effectiveness in detecting and alarming B\&D events, which coincides with the apnea, and resolving it by providing foot stimulation [10]. ApneBoot not only resolves apneas but also aims to terminate episodes of prolonged hypoxia which are proven to cause injury to preterm babies [11]. To further validate this hypothesis another study is ongoing on a larger sample size. The ongoing study is an RCT, being conducted at 2 large centers 1 public \& private hospital, on a total of 76 babies. The primary objective of the study is to compare the duration of hypoxia in each baby during the study period. Among the 40 babies enrolled to date at both the sites, results look promising. It is observed that the intervention group has lower mean hypoxia duration compared to that of control group in the neonate population recruited. Based on the published and ongoing studies, ApneBoot was found to be accurate at detecting bradycardia \& hypoxia events related to apnea. Further, it was also reported to safely stimulate a newborn to resolve these apnea episodes.

The next step will be to look into the potential use of ApneBoot in transport and humanitarian settings due to its reusability and portability. Our future study is being designed to observe and report feedback from users (nurses and doctors) of the device. We also aim to quantify the reduction in workload of SNCU nurses for apnea management before and after ApneBoot use.

\section{Impact to Date \& Future Plans}

ApneBoot's primary beneficiaries are the subset of 15 million premature newborns in India and globally receiving care in lowresource settings like the Special Newborn Care Units (SNCUs) in government hospitals as well as Primary Health Centres and Community Health Centers. The publication and ongoing studies on the device has generated visibility and appeal in Government Medical Colleges and private hospitals all over India. To date 25 hospitals all over India reaching over 200 preterm newborns ( $\sim 80 \%$ from lower socioeconomic population) have used the device. One government trial at Calicut Medical College has resulted in a NHM recommendation letter for ApneBoot use. BEMPU plans to monitor future deployments of the device as part of an impact and feasibility evaluation to collect evidence that can be used to drive further adoption.

\section{Conclusion}

The need for a device that helps in managing Apnea of Prematurity (AOP) was realized to reduce the burden of morbidity and mortality in the infant population. ApneBoot is a novel device in the market that not only detects and alerts for apnea but also resolves the episodes by tactile auto-stimulation. A recent clinical study reports the accuracy of the device to be $70 \%$, demonstrating its potential in apnea management especially in low resource settings.

\section{Conflict of Interest Statement}

No potential conflict of interest is reported by the authors. The authors alone are responsible for the content and writing of the paper.

\section{Financial Support Statement}

ApneBoot received a SEED grant from Saving Lives at Birth program - A partnership between the United States Agency for 
International Development (USAID), the Norwegian Ministry of Foreign Affairs, the Bill \& Melinda Gates Foundation, Grand Challenges Canada, the Department for International Development, United Kingdom of Great Britain and Northern Ireland (DFID), and the Korea International Cooperation Agency (KOICA)

\section{Acknowledgment}

We sincerely thank Dr. Saudhamini Nesargi, St. John's Hospital, Bangalore; Dr. Himabindu Singh, Dr. Alimelu Rao, Dr. Swapna Lingaldinna, Nilofer Hospital, Hyderabad for all their valuable clinical inputs. We would like to acknowledge the nursing officer's from St. John's hospital, Bangalore and Nilofer Hospital, Hyderabad for their incredible support through the development of the device. We also appreciate the contribution of Dr. Vinita V. Khot, Bempu Health, Bangalore, towards the writing of this paper.

\section{References}

1. Blencowe H, Simon C, Oestergaard M, Chou D, Moller A, et al. (2012) National, regional, and worldwide estimates of preterm birth rates in the year 2010 with time trends since 1990 for selected countries: A systematic analysis and implications. The lancet 379(9832): 2162-2172.

2. Barrington K, Finer N (1991) The natural history of the appearance of apnea of prematurity. Pediatric research 29(4): 372-375.

ISSN: 2574-1241

DOI: $10.26717 /$ BJSTR.2020.27.004530

Mona Sharma. Biomed J Sci \& Tech Res

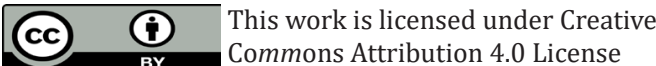

Submission Link: https://biomedres.us/submit-manuscript.php
3. Eichenwald C (2016) Apnea of prematurity. Pediatrics 137(1): e20153757.

4. Fairchild K, Mohr M, Paget Brown A, Tabacaru C, Lake D, et al. (2016) Clinical associations of immature breathing in preterm infants: Part 1-central apnea. Pediatric research 80(1): 21-27.

5. Zhao J, Gonzalez F, Mu D (2011) Apnea of prematurity: From cause to treatment. European journal of pediatrics 170(9): 1097-1105.

6. Cramer JE, Dekker J, Dankelman J, Pauws SC, Hooper SB, et al. (2018) Effect of tactile stimulation on termination and prevention of apnea of prematurity: A systematic review. Frontiers in pediatrics 6: 45 .

7. Neogi SB, Khanna R, Chauhan M, Sharma J, Gupta G, et al. (2016) Inpatient care of small and sick newborns in healthcare facilities. Journal of Perinatology 36(3): S18-S23.

8. McClure C, Jang SY, Fairchild K (2016) Alarms, oxygen saturations, and $\mathrm{SpO}_{2}$ averaging time in the NICU. Journal of neonatal perinatal medicine 9(4): 357-362.

9. https://www.nonin.com/support/oem3

10. Lingaldinna S, Singh H, Sharma M (2020) Efficacy of a novel device to detect, alert and resolve neonatal apnea- Pilot study. Innovative Journal of Medical \& Health Sciences.

11. Poets Christian F, Roberts RS, Schmidt B, Whyte RK, Asztalos EV, et al. (2015) Association between intermittent hypoxemia or bradycardia and late death or disability in extremely preterm infants. Jama 314(6): 595603. 